\title{
A Comparison between the Outcomes of Direct Anterior and Posterior Approaches for Rates of Dislocation and Infection in Primary Total Hip Arthroplasty
}

\author{
Zohair M. Ahmed', Hamid A. Mahmud ${ }^{2 *}$, Imad M. Rasul ${ }^{3}$ \\ ${ }^{1}$ Department of Orthopedic Surgery, Erbil Teaching Hospital, Erbil, Kurdistan Region, Iraq, ${ }^{2}$ Department of Orthopedic Surgery, Sulaymaniyah \\ Teaching Hospital, Sulaymaniyah, Kurdistan Region, Iraq, ${ }^{3}$ Department of Orthopedic Surgery, Erbil Teaching Hospital, Erbil, Kurdistan \\ Region, Iraq
}

\section{${ }^{*}$ Corresponding author: \\ Hamid A. Mahmud, \\ Department of Orthopedic \\ Surgery, Sulaymaniyah \\ Teaching Hospital, \\ Sulaymaniyah, Kurdistan \\ Region, Iraq. E-mail: hamed. \\ mahmood@univsul.edu.iq}

Received: 10 January 2018

Accepted: 04 September 2018

Published: 30 June 2020

DOI

10.25156/pt.v10n1y2020.pp194-199

\section{A B S T R A C T}

Background: Direct anterior hip replacement is a slightly aggressive surgical procedure but potentially widespread. It involves opening on the front of the hip to allow the joint to be substituted by moving muscles aside along their ordinary tissue planes without removing any tendons. Nevertheless, there is diminutive consent concerning the threats and profits of this method compared to the posterior approach (PA) regarding the rates of dislocation and infection. Methods: This research is a prospective study conducted in Erbil city from September 2015 to September 2017 on 57 patients, including 28 females and 29 males, with primary total hip arthroplasty (THA). Twenty-nine patients were enrolled in the direct anterior approach (DAA: First group), and 28 patients were registered in the PA group (PA: Second group). Their age ranged between 49 and 80 years, and the regular follow-up was 12 months for all patients. Results: It was found that using the DAA, there was a significantly $(P \leq 0.05)$ lower incidence rate of dislocation in patients undergoing THA than the PA. No significant difference in the rates of infection was noticed between both groups. However, slightly shorter operational time and extent of the incision were noticed in the DAA group, and less blood loss was found in the PA group (non-significant). Conclusion: We realized that DAA has a clear advantage over PA regarding the lower incidence in the dislocation risk and without variance in the infection rates.

Keywords: Hip prosthesis; Direct anterior and posterior approaches; Prospective study; Orthopedic surgery

\section{INTRODUCTION}

Total hip arthroplasty (THA) is considerable thought to be a standout among the best orthopedic surgeries. The lion's share of patients experiencing THA encounter assistance with discomfort, prolonged portability, improved capacity, and upgrading of personal satisfaction from previously weakening joint illness (Barrett et al., 2013; Restrepo et al., 2010). A small number of THA patients continue experiencing manifestations, utmost regular pain, which keep their arrival to occupied ability and action. Conceivable leads to instability, fixation failure, and impairment to delicate tissues related to the operation area's injury. The selection of the perfect cautious method can bound these dangers and therefore enhances the consequence of THA (Bremer et al., 2011).

Standard operating methodologies in THA comprise anterior, anterolateral, direct lateral, trans-trochanteric, and posterior approaches (PA) (Knight et al., 2011;
Varacallo et al., 2020). Previously, endeavors to enhance conventional careful methods concentrated on limiting the surgical incision distance. Hence, various minimally invasive surgery practices were designed, such as the posterolateral technique (Bergin et al., 2011), the double-incision technique, using Smith-Petersen anterior approach joint with a posterior slit (Lichstein et al., 2018; Ricci et al., 2018), with mini-posterior and minianterolateral operating methods (Berstock et al., 2014), and a modified anterolateral Watson-Jones approach (Chen and Berger, 2013). Gradually, it was realized that the length of the incision is not significant to the surgical outcome than soft-tissue blood supply impairment, innervations, muscle rupture, and bone protection (Miller et al., 2018).

The Smith-Peterson anterior approach is collectively covered the direct anterior approach (DAA) (Ricci et al., 2018). Followers found that the DAA results in minor trauma to the soft tissue than other surgical methods. In 
addition, it tracks internervous and intermuscular positions, especially the anatomic space connecting the tensor fasciae latae and sartorius muscles (Khan et al., 2017; Meermans et al., 2017). The conservation of muscle influences to bone and the prevention of separation of muscle tissue propose the feasibility for enhanced exclusive hip constancy and reduced threat of hip displacement after the process (Post et al., 2014).

Various reviews of observational examinations give a solid premise for the utilization of the DAA. In this respect, a cohort study on 1152 patients was conducted by the anterior THA collaborative investigators using a single-incision DAA. The researchers realized an excellent impediment profile, prompt reappearance to function, and a drop in obstacles in surgeons with $>100$ case practices (Bhandari et al., 2009). Another retrospective study that compared the DAA to the transgluteal approach utilizing MRI assessment; determined that the DAA displayed an enhanced soft-tissue recovery (Bremer et al., 2011).

In addition, some other researches that correlated the DAA with different surgical methodologies exhibited superior results for DAA, including enhanced perioperative outcome, early utilitarian recuperation, and discomfort switch (Barrett et al., 2013; Goebel et al., 2012; Meena, 2012). At the same time, some studies stated complication issues with DAA (Bhadra et al., 2012; Spaans et al., 2012).

In the United States of America (USA), the commonly applied approach for THA is PA despite more popularity and fast generalization of the DAA. It has professed enhancements in initial functional retrieval and diminished displacement rates, with a few experts forceful for no hip care actions after the surgery. Main profits are fewer softtissue damage and more fast healing while maintaining a lesser displacement hazard related to anterior techniques (Maratt et al., 2016).

As utmost research indicates little distinction in functional recuperation past a month and a half between the two approaches, dislocation hazard remains one of the primary explanations behind pushing the anterior approach. However, there is an excellent rate of impassiveness from injury to the lateral femoral cutaneous nerve, notwithstanding a possibly general advanced danger of difficulties amid a specialist's expectation to learn and adapt (Cheng et al., 2017; Christensen and Jacobs, 2015; Malek et al., 2016). Therefore, this study aimed to check the effect of THA through DAA and PA in patients to indicate which one results in a negligible risk of dislocation and infection at early follow-ups.

\section{MATERIALS AND METHODS}

\section{Study Area}

Both DAA and the PA techniques were conducted in Zheen Private Hospital, Erbil city, by an expert single orthopedic surgeon between September 2015 and September 2017.

\section{Patients}

Fifty-seven patients were randomly allocated into DAA and PA groups, in which 29 of them were in the first group and 28 were in the second group.

\section{Inclusion Criteria}

Patients that necessitate a non-cemented, primary THA for osteoarthritis, avascular necrosis, and femoral neck fractures caused by trauma were included in this study.

\section{Exclusion Criteria}

Patient with a history of active sepsis in the joint, allergy to any component of the study device, or those had a total or partial absence of the muscular or ligamentous apparatus, or those known to have moderate to severe renal insufficiency, vascular insufficiency, muscular atrophy, or neuromuscular disease in either leg were excluded from this study.

\section{Ethical Approval}

The Institute Review Board approval for this prospective, non-randomized clinical study was obtained from the Directorate of Health, Erbil city. In addition, the complete and proper information about the study was explained to each participant before the commencement of the surgery, as well as signed patient consent forms, was collected from all of them.

\section{Operational Protocol}

Standardized operative protocols comprising pain management and rapid rehabilitation were employed in this study. Regarding the range of motion, the first group did not have any restrictions, while the second group had a repetitive range of motion restrictions.

For the first group, a modern fracture table with direct visualization was used in which the patient was positioned supine wearing a pair of boots to stable a posture. Then, an anterior skin incision $(10-14 \mathrm{~cm})$ was made, and an intermuscular plane was used to enter the anterior hip capsule. Later on, through the anteriorly opened hip capsule, a femoral neck osteotomy was conducted that followed by the removal of the femoral head, and the acetabular retractors were located with reaming of the acetabulum. Consequently, a hydraulic trochanteric hook was used to elevate the proximal femur. Next, the femoral canal was broached to the 
proper size, then the extent and offset of the wound were tested. Finally, the prostheses were entered with press-fit fixation and closed regularly.

Regarding the PA technique, a standard operating room table was used, and the patients were positioned on the lateral decubitus site. Then, a 10-14 cm skin incision was made on the posterior-lateral corner of the hip; the gluteus maximus muscle was cut parallel to its fibers, followed by an opening of the short external rotators and posterior capsule. Later on, the hip was displaced posteriorly, and a femoral neck osteotomy was performed followed by the insertion of the acetabular and femoral components with press-fit fixation.

Instantly, the patients were checked after 1.5, 3, 6, and 12 months post-operative for assessing the rate of dislocation and infection. In addition, conventional anteroposterior pelvis and cross-table lateral radiographs were obtained $24 \mathrm{~h}$ after the operation and 1.5, 3, 6, and 12 months post-operative. The radiographs of 1.5 months were observed to find cup orientations, including angles of inclination and anteversion, and stem orientation, then graded as valgus, neutral, or varus. At the same time, the radiographs of 12 months were checked to find skeletal fixation.

\section{Patient Rehabilitation}

Patients in both groups were exposed to similar preoperative, perioperative, anesthetic, rehabilitation, and pain protocols. Patients received prophylactic antibiotics (ceftriaxone vial $1.0 \mathrm{~g} / 1 \mathrm{~h}$ before operation followed by once a day for days after the procedure). The pain protocol in this study consisted of premedication before the commencement of the process followed by post-operative oral narcotic and non-narcotic treatment (when required) and intravenous narcotics for advanced pain. Patients were received a subcutaneous Clexane (enoxaparin) vial (4000 IU) once a day/2 weeks to prevent venous thromboembolism.

In addition, patients practiced physiotherapy $24 \mathrm{~h}$ after the operation (twice a day), started from using a frame walker then progressed to using of non-assistive device that was accepted by the patient. Finally, we decided to discharge the patients when they could mobilize without harm, did not complain about pain, and were medically stable. However, the patients were followed up, usually after 2 and 6 weeks post-discharge.

\section{Statistical Analysis}

All collected data were entered into SPSS software version 25 (IBM, USA), and the statistically significant value $(\mathrm{P})$ was set at 0.05 .

\section{RESULTS}

Primary total hip arthroplasties were done on patients in which 29 of them underwent DAA and 28 underwent PA, with complete follow-up. Table 1 shows the patient's sociodemographic data (gender, age, and body mass index $[\mathrm{BMI}])$ in both groups and other parameters, including operative side and pre-operative diagnosis. From the table below, we realized that females were 28 and males were 29, age ranged between 49 and 80 with a mean range of 61.79 \pm 23.03 . Regarding the operative side, the operation was done on the left side in the majority of the patients (33 out of 57), while the operation was done on the right side in 24 out of 57 patients. On the other hand, the majority of the patients were diagnosed with osteoarthritis (37 out of 57 ), followed by trauma (11 out of 57), and then vascular necrosis in nine cases. No significant differences were seen among both groups for all parameters.

In addition, the intraoperative data were comparable between both groups regarding anesthesia type, operation time, intraoperative blood loss, the extent of the incision, and length of stay in the hospital. In a total of 57 cases, only 18 cases underwent spinal anesthesia, while the rest (majority: 39 patients) underwent general anesthesia. The operation process took more time in the PA group (63 min) than in the DAA group (55 min). Collectively, no significant difference was observed between both groups for all studied parameters; however, slightly shorter operative time, less blood loss, reduced length of the incision, and less duration of stay in hospital were noticed in the DAA group [Table 2].

Radiographs were taken to patients on the $1^{\text {st }}$ post-operative day, and the amount of cup inclination and anteversion or retroversion angles were measured. No significant differences were noticed for all studied characteristics

Table 1: Sociodemographic and some baseline clinical characteristics of the research population

\begin{tabular}{lccc}
\hline Characteristic & $\begin{array}{c}\text { DAA group } \\
(\boldsymbol{n}=29)\end{array}$ & $\begin{array}{c}\text { PA group } \\
(\boldsymbol{n}=28)\end{array}$ & P value \\
Gender & & & \\
$\quad$ Female & $15(51.7 \%)$ & $13(46.4 \%)$ & 0.087 \\
$\quad$ Male & $14(48.2 \%)$ & $15(53.6 \%)$ & \\
Age (years) & $49-80$ & $49-80$ & 0.092 \\
Mean \pm SD & $62.06 \pm 8.8$ & $61.35 \pm 7.72$ & \\
Body mass index $\left(\mathrm{Kg} / \mathrm{m}^{2}\right)$ & $29.3 \pm 5.4$ & $28.7 \pm 5.9$ & 0.171 \\
Operation side & & & \\
$\quad$ Right & $13(44.8 \%)$ & $11(39.2 \%)$ & 0.13 \\
$\quad$ Left & $16(55.2 \%)$ & $17(60.8 \%)$ & \\
Pre-operative diagnosis & & & \\
$\quad$ Osteoarthritis & $19(65.5 \%)$ & $18(64.3 \%)$ & 0.087 \\
$\quad$ Vascular necrosis & $6(20.7 \%)$ & $3(10.7 \%)$ & \\
$\quad$ Trauma & $4(13.8 \%)$ & $7(25 \%)$ & \\
\hline
\end{tabular}


between both groups. All patients underwent the same rehabilitation protocol, and their restrictions were clarified (data not shown).

Regarding the comparison of the rate of dislocations and infection between DAA and PA groups, out of 29 patients in the first group, none of them had a dislocation. Still, one case had an infection 1 year after the operation. In the PA group, four patients had a dislocation despite good cup inclination and anteversion, which was statistically significant $(\mathrm{P}=0.043)$, and only one case had an infection [Table 3].

\section{DISCUSSION}

THA is cost effective and constantly successful surgeries implemented in orthopedics. It offers consistent consequences for patients having advanced degenerative hip osteoarthritis, pain numbness, functional restoration, and enhanced quality of life. In this respect, DAA and PA were developed using the short Smith-Peterson approach, which gave adequate acclaim and excellent clinical outcomes (Huang et al., 2012).

In this research, there was no significant difference for sociodemographic data (sex, age, and BMI) and operative side and pre-operative diagnosis between both groups. These outcomes are in agreement with a study outcome of a recently conducted research in the USA (Maldonado et al., 2019), while it is in contrast to another study outcomes (Tsukada and Wakui, 2015). Instantly, old age, BMI $>30 \mathrm{~kg} / \mathrm{m}^{2}$, surgeon skill, and femoral head size

Table 2: Studied parameters of surgical operation and hospitalization

\begin{tabular}{lccc}
\hline Characteristic & $\begin{array}{c}\text { DAA group } \\
(\boldsymbol{n}=29)\end{array}$ & $\begin{array}{c}\text { PA group } \\
(\boldsymbol{n}=\mathbf{2 8})\end{array}$ & $\boldsymbol{P}$ value \\
\hline Anesthesia & & & \\
$\quad$ General & $19(65.5 \%)$ & $20(71.4 \%)$ & 0.145 \\
Spinal & $10(34.5 \%)$ & $8(28.6 \%)$ & \\
Operation time (minute) & $55.35 \pm 8.59$ & $63.21 \pm 8.41$ & 0.141 \\
Intraoperative blood & $368.9 \pm 7.84$ & $383.92 \pm 1.62$ & 0.404 \\
loss (mL) & & & \\
Incision length (cm) & $10.1 \pm 1.42$ & $11.76 \pm 1.78$ & 0.46 \\
Length of stay (Day) & $2.8 \pm 0.93$ & $2.9 \pm 0.08$ & 0.094 \\
\hline
\end{tabular}

DAA: Direct anterior approach, PA: Posterior approach

Table 3: Comparison of the complication rates between DAA and PA groups

\begin{tabular}{lccc} 
Characteristic & $\begin{array}{c}\text { DAA group } \\
(\boldsymbol{n}=29)\end{array}$ & $\begin{array}{c}\text { PA group } \\
(\boldsymbol{n = 2 8 )})\end{array}$ & $\boldsymbol{P}$ value \\
Cup inclination & $43.1 \pm 5.2$ & $42.7 \pm 6.7$ & 0.089 \\
Cup anteversion & $21.3 \pm 4.8$ & $23.7 \pm 7.2$ & 0.094 \\
Dislocation & $0(0 \%)$ & $4(14.28 \%)$ & $0.043^{*}$ \\
Infection & $1(3.44 \%)$ & $1(3.44 \%)$ & 0.987 \\
\hline
\end{tabular}

DAA: Direct anterior approach, PA: Posterior approach impact dislocation rates after THA, while sex does not affect dislocation rate (Rowan et al., 2018).

On the other hand, we found a slightly lower rate of blood loss, operation time, less hospital stay, and earlier return to activities in the DAA group than the PA group. These results were not statistically significant and needed further study to confirm the benefits of DAA in THA. These outcomes are similar to those found by other surgeons (Barrett et al., 2013; Martin et al., 2013).

In general, DAA is an internervous plane method between the regions of innervation of the femoral nerve medially and the inferior and superior gluteal nerves laterally. In DAA, the basis of the tensor fascia latae, sartorius, and rectus femoris is not separated, and additions of the gluteus medius and minimus muscle are not disconnected by the unsharpened revelation process. Thus, DAA is a muscle protecting technique that leads to a rapid, efficient recovery. This hypothesis is confirmed by some surgeons in different countries, including our study (Cheng et al., 2017; Malek et al., 2016; Tsukada and Wakui, 2015; Zawadsky et al., 2014).

In this study, there were no significant differences between DAA and PA when conducting primary THA in terms of dislocation rate, which is suggested mainly to be associated with surgeon experience and preference, supposed negativity and positivity of each technique, patient desire to choose the approach. This finding is similar to the outcomes of other surgeons using the same processes in THA (Maratt et al., 2016; Tamaki et al., 2016; Tripuraneni et al., 2016). In contrast, a significantly lower rate of dislocation was found in THD through DAA than PA (Tsukada and Wakui, 2015). Expert surgeons suggested that navigation, robotics, lipped liners, and dual-mobility acetabular components may improve dislocation rates as well as risks for dislocation, and measures to mitigate the risk should be determined (Rowan et al., 2018).

In general, DAA is not commonly conducted to patients, but its faster recovery and reduced dislocation rate made the DAA become more popular than PA and a muscle-sparing process, although some degrees of complication and risk of early revision to DAA were observed (Malek et al., 2016). However, the high familiarity of PA to surgeons makes it to be somewhat popular in spite of its protection of the hip abduction, which avoids the development of a limp and simplicity of extension for complicated or revised cases (Radoicic et al., 2018).

In the current study, there was no significant difference between both groups regarding infection rates, and this result is agreed with outcomes obtained by other surgeons (De Geest et al., 2013; Poehling-Monaghan et al., 2015) 
who studied the rate of infection in hip arthroplasties. However, a group of researchers in the USA reported a significant prevalence of wound complications on THA performed through DAA and PA, especially the first one that suggested to be reoperated back (Christensen et al., 2014). Similarly, significantly increased rates of postoperative infection were found in severely obese patients after the DAA process (Purcell et al., 2016).

The main limitation of this study includes not randomly assigning the patients to treatment choices. Hence, inherent variances in prognosis might affect the surgeon's preference to use any assumed technique available and adorable.

\section{CONCLUSION}

We concluded that the performance of THA using DAA exhibits less dislocation rate and is more suitable than PA. However, there was no significant difference in the risk of infection between both techniques. Therefore, we recommend modifying the PA technique better, including utilization of posterior capsular repair, improved offset stems, and more excellent femoral head to diminish the rate of dislocation.

\section{ACKNOWLEDGMENTS}

The authors appreciate the staff of the Department of Orthopedic Surgery, Zheen Private Hospital, Erbil city, for their assistance and support to this study.

\section{CONFLICTS OF INTEREST}

No conflicts of interest to this research study are reported by the authors. The research is entirely self-funded, and no specific fund or grant was obtained to perform it.

\section{REFERENCES}

Barrett, W.P., Turner, S.E. and Leopold, J.P. 2013. Prospective randomized study of direct anterior vs postero-lateral approach for total hip arthroplasty. J. Arthroplast. 28: 1634-1638.

Bergin, P.F., Doppelt, J.D., Kephart, C.J., Benke, M.T., Graeter, J.H., Holmes, A.S., Haleem-Smith, H., Tuan, R.S. and Unger, A.S. 2011. Comparison of minimally invasive direct anterior versus posterior total hip arthroplasty based on inflammation and muscle damage markers. J. Bone Joint Surg. 93: 1392-1398.

Berstock, J.R., Blom, A.W. and Beswick, A.D. 2014. A systematic review and meta-analysis of the standard versus mini-incision posterior approach to total hip arthroplasty. J. Arthroplast. 29: 1970-1982.

Bhadra, A., Yakkanti, M. and Malkani, A. 2012. Primary Total Hip Arthroplasty Using a Direct Anterior vs. Posterolateral Approach: A comparative study. Annual Meeting American Academy of
Orthopedic Surgeons, San Francisco, California.

Bhandari, M., Matta, J. and Dodgin, D. 2009. Anterior total hip arthroplasty collaborative investigators outcomes following the single incision anterior approach to total hip arthroplasty: A multicenter observational study. Orthop. Clin. North Am. 40: 329-342.

Bremer, A., Kalberer, F., Pfirrmann, C. and Dora, C. 2011. Soft-tissue changes in hip abductor muscles and tendons after total hip replacement: Comparison between the direct anterior and the transgluteal approaches. J. Bone Joint Surg. 93: 886-889.

Chen, D. and Berger, R.A. 2013. Outpatient minimally invasive total hip arthroplasty via a modified Watson-Jones approach: Technique and results. Instr. Course Lect. 62: 229-236.

Cheng, T.E., Wallis, J.A., Taylor, N.F., Holden, C.T., Marks, P., Smith, C.L., Armstrong, M.S. and Singh, P.J. 2017. A prospective randomized clinical trial in total hip arthroplasty comparing early results between the direct anterior approach and the posterior approach. J Arthroplast. 32: 883-890.

Christensen, C. and Jacobs, C. 2015. Comparison of patient function during the first six weeks after direct anterior or posterior total hip arthroplasty (THA): A randomized study. The J. Asrthroplast. 30: 94-97.

Christensen, C., Karthikeyan, T. and Jacobs, C. 2014. Greater prevalence of wound complications requiring reoperation with direct anterior approach total hip arthroplasty. J. Arthroplast. 29: 1839-1841.

De Geest, T., Vansintjan, P., De Loore, G. 2013. Direct anterior total hip arthroplasty: complications and early outcome in a series of 300 cases. Acta Orthop Belgica. 79: 166-173.

Goebel, S., Steinert, A.F., Schillinger, J., Eulert, J., Broscheit, J., Rudert, M. and Nöth, U. 2012. Reduced postoperative pain in total hip arthroplasty after minimal-invasive anterior approach. Int. Orthop. 36: 491-498.

Huang, X., Cheng, L., He, T., Liu, Y., Wang, Y. and Zhu, H. 2012. Efficiency of minimally invasive total hip arthroplasty with anterolateral approach versus the traditional total hip arthroplasty with posterolateral approach. Chin. J. Tissue Eng. Res. 16: 1538-1542.

Khan, O., Malviya, A., Subramanian, P., Agolley, D. and Witt, J. 2017. Minimally invasive periacetabular osteotomy using a modified Smith-Petersen approach: Technique and early outcomes. Bone Joint J. 99: 22-28.

Knight, S.R., Aujla, R. and Biswas, S.P. 2011. Total hip arthroplastyover 100 years of operative history. Orthop. Rev. 3: e16.

Lichstein, P.M., Kleimeyer, J.P., Githens, M., Vorhies, J.S., Gardner, M.J., Bellino, M. and Bishop, J. 2018. Does the WatsonJones or modified Smith-Petersen approach provide superior exposure for femoral neck fracture fixation? Clin. Orthop. Relat. Res. 476: 1468-1476.

Maldonado, D.R., Laseter, J.R., Kyin, C., Lall, A.C. and Domb, B.G. 2019. Direct anterior approach in total hip arthroplasty leads to superior outcomes at 3-month follow-up when compared with the posterior approach: A matched study using propensity score analysis. JAAOS Glob. Res. Rev. 3: 1-7.

Malek, I., Royce, G., Bhatti, S., Whittaker, J., Phillips, S., Wilson, I., Wootton, J. and Starks, I. 2016. A comparison between the direct anterior and posterior approaches for total hip arthroplasty: The role of an 'Enhanced Recovery'pathway. Bone Joint J. 98: 754-760.

Maratt, J.D., Gagnier, J.J., Butler, P.D., Hallstrom, B.R., Urquhart, A.G. and Roberts, K.C. 2016. No difference in dislocation seen in anterior vs posterior approach total hip arthroplasty. J. Arthroplast. 31: 127-130. 
Martin, C.T., Pugely, A.J., Gao, Y. and Clark, C.R. 2013. A comparison of hospital length of stay and short-term morbidity between the anterior and the posterior approaches to total hip arthroplasty. J. Arthroplast. 28: 849-854.

Meena, S. 2012. Comparison of primary total hip replacements performed with a direct anterior approach versus the standard lateral approach: Perioperative findings. J. Orthop. Traumatol. 13: $115-117$.

Meermans, G., Konan, S., Das, R., Volpin, A. and Haddad, F. 2017. The direct anterior approach in total hip arthroplasty: A systematic review of the literature. Bone Joint J. 99: 732-740.

Miller, L.E., Gondusky, J.S., Bhattacharyya, S., Kamath, A.F., Boettner, F. and Wright, J. 2018. Does surgical approach affect outcomes in total hip arthroplasty through 90 days of followup? A systematic review with meta-analysis. J. Arthroplast. 33: 1296-1302.

Poehling-Monaghan, K.L., Kamath, A.F., Taunton, M.J. and Pagnano, M.W. 2015. Direct anterior versus miniposterior THA with the same advanced perioperative protocols: Surprising early clinical results. Clin. Orthop. Relat. Res. 473: 623-631.

Post, Z.D., Orozco, F., Diaz-Ledezma, C., Hozack, W.J. and Ong, A. 2014. Direct anterior approach for total hip arthroplasty: Indications, technique, and results. J. Am. Acad. Orthop. Surg. 22: 595-603.

Purcell, R.L., Parks, N.L., Gargiulo, J.M. and Hamilton, W.G. 2016. Severely obese patients have a higher risk of infection after direct anterior approach total hip arthroplasty. J. Arthroplast. 31: 162-165.

Radoicic, D., Zec, V., Elassuity, W.I. and Azab, M.A. 2018. Patient's perspective on direct anterior versus posterior approach total hip arthroplasty. Int. Orthop. 42: 2771-2775.

Restrepo, C., Parvizi, J., Pour, A.E. and Hozack, W.J. 2010.
Prospective randomized study of two surgical approaches for total hip arthroplasty. J. Arthroplast. 25: 671-679. e671.

Ricci, W.M., McAndrew, C.M., Miller, A.N. and Avery, M.C. 2018. Open reduction and internal fixation of the femoral head via the Smith-Petersen approach. J. Orthop. Trauma. 32: S16-S17.

Rowan, F.E., Benjamin, B., Pietrak, J.R. and Haddad, F.S. 2018. Prevention of dislocation after total hip arthroplasty. J. Arthroplast. 33: 1316-1324.

Spaans, A.J., Hout, J.A. and Bolder, S.B. 2012. High complication rate in the early experience of minimally invasive total hip arthroplasty by the direct anterior approach. Acta Orthop. 83: 342-346.

Tamaki, T., Oinuma, K., Miura, Y., Higashi, H., Kaneyama, R. and Shiratsuchi, H. 2016. Epidemiology of dislocation following direct anterior total hip arthroplasty: A minimum 5-year follow-up study. J. Arthroplast. 31: 2886-2888.

Tripuraneni, K.R., Munson, N.R., Archibeck, M.J. and Carothers, J.T. 2016. Acetabular abduction and dislocations in direct anterior vs posterior total hip arthroplasty: A retrospective, matched cohort study. J. Arthroplast. 31: 2299-2302.

Tsukada, S. and Wakui, M. 2015. Lower dislocation rate following total hip arthroplasty via direct anterior approach than via posterior approach: Five-year-average follow-up results. Open Orthop. J. 9: 157-162.

Varacallo, M., Luo, T.D. and Johanson, N.A. 2020. Total Hip Arthroplasty. StatPearls Publishing, Techniques Treasure Island, FL.

Zawadsky, M.W., Paulus, M.C., Murray, P.J. and Johansen, M.A. 2014. Early outcome comparison between the direct anterior approach and the mini-incision posterior approach for primary total hip arthroplasty: 150 consecutive cases. J. Arthroplast. 29: 1256-1260. 